\title{
Viewpoint \\ New insights into the biological function of BRCA2 from its structural interactions
}

\author{
Alison Waterworth \\ Molecular Medicine Unit, Clinical Sciences Building, St James' University Hospital, Leeds, UK \\ Corresponding author: Alison Waterworth (e-mail: alisonwaterworth@doctors.org.uk) \\ Published: 4 February 2003
}

Breast Cancer Res 2003, 5:107-108 (DOI 10.1186/bcr581)

(C) 2003 BioMed Central Ltd (Print ISSN 1465-5411; Online ISSN 1465-542X)

\section{Introduction}

Around $50 \%$ of all familial breast and ovarian cancers are due to mutations in BRCA1 and BRCA2. Germline mutations in the BRCA2 gene are associated with an increased susceptibility to breast cancer (in both males and females) and they also confer an increased risk of early onset ovarian, prostate, and pancreatic cancer. The biological function of BRCA2 in the cell is still uncertain, although there is increasing evidence for a role in the repair of DNA by homologous recombination. BRCA2 and RAD51 (a homolog of the baterial recombination protein RecA) both co-localise to nuclear foci thought to be sites of DNA damage and repair and these nuclear foci fail to form in BRCA2 deficient cells. Loss of BRCA2 leads to error prone repair of double strand DNA breaks and in dividing cells can lead to chromosomal abberations and loss of genetic information. Compelling evidence of a more direct role for BRCA2 in DNA repair is provided by two recent studies investigating some of the protein's structural interactions.

\section{Structural interactions of BRCA2}

In an exceptional publication in Science [1], Yang and colleagues demonstrate the crystal structure of the 800residue carboxyl-terminal domain of BRCA2 that lies beyond the sequence of highly conserved BRC motifs (sets of amino acid repeats in the centre of BRCA2). This carboxyl-terminal region is likely to play an important role in the tumour suppressor function of BRCA2 as it corresponds to the most conserved portion of BRCA2 across different species and contains $27 \%$ of tumour-derived missense mutations. The authors strengthen this hypothesis by presenting the 3.1 angstrom structure of the carboxyl-terminal domain bound to another protein, DSS1 (deleted in split hand/split foot syndrome), and show that this structure contains multiple areas similar to single stranded and double stranded DNA binding motifs. They also show that this domain of BRCA2 can bind to single stranded DNA and stimulate RAD51 mediated recombination in vitro. Their data thus indicate a direct role for
BRCA2 in homologous recombination and provide a structural insight into the loss of recombination mediated DNA repair in BRCA2 associated cancers.

Pellegrini and colleagues from Cambridge University explore further the structural interactions between BRCA2 and RAD51 and their role in DNA repair [2]. This paper reports for the first time the crystal structure of the complex between a BRC repeat and the catalytic domain of RAD51. The BRC repeat is found to mimic a motif in RAD51 that serves as an interface between adjacent RAD51 monomers. Through molecular mimicry, BRCA2 may therefore control the formation of the RAD51 nucleoprotein filament, which is essential for DNA recombination. This provides a structural basis for the BRCA2 regulation of RAD51 function and explains how BRCA2 mutations may disrupt the predicted interactions between $B R C$ and RAD51 and cause increased cancer susceptibility.

\section{Conclusion}

Both studies outlined demonstrate the importance of structural analyses to further knowledge of protein function. They provide direct evidence for the role of BRCA2 in DNA repair and also a structural basis for the loss of recombination-mediated double strand DNA break repair in BRCA2-associated cancers. The challenge now is to take advantage of this deficiency in homologous recombination to specifically target and destroy BRCA2 mutant cells in cancer patients.

\section{Competing interests}

None declared.

\section{References}

1. Yang $H$, Jeffrey $P D$, Miller J, Kinnucan E, Sun $Y$, Thoma NH, Zheng N, Chen PL, Lee WH, Pavletich NP: BRCA2 function in DNA binding and recombination from a BRCA2-DSS1-sSDNA structure. Science 2002, 297:1837-1848.

2. Pellegrini L, Yu DS, Lo T, Anand S, Lee M, Blundell TL, Venkitaraman AR: Insights into DNA recombination from the structure of a RAD51-BRCA2 complex. Nature 2002, 420: 287-293. 


\section{Note}

This article is based on papers highlighted by Faculty of 1000 (http://www.facultyof1000.com/start.asp), a web-based literature awareness service. Faculty of 1000 evaluations available for articles cited in this report may be viewed at: http://breast-cancer-research.com/reports/bcr581.asp

\section{Correspondence}

Alison Waterworth, Molecular Medicine Unit, Clinical Sciences Build-

ing, St James' University Hospital, Leeds LS9 7TF, UK. Tel: +44

(0)113 206 6655; fax: +44 (0)113 244 4475; e-mail: alisonwaterworth@doctors.org.uk 